\title{
Erratum to: Probing Protein Kinase-ATP Interactions Using a Fluorescent ATP Analog
}

\section{Leslie E.W. LaConte, Sarika Srivastava, and Konark Mukherjee}

\section{Erratum to:}

Chapter 11 in: Iulia M. Lazar et al. (eds.), Proteomics for Drug Discovery: Methods and Protocols, Methods in Molecular Biology, vol. 1647, https://doi.org/10.1007/978-1-4939-7201-2_11

When the volume originally published the author neglected to acknowledge the laboratory that funded the research. The updated chapter now contains that information. 